(e-migrinter)

e-Migrinter

12 | 2014

Les chiffres de l'immigration : un regard critique sur les statistiques

\title{
Carine Pina-Guerassimoff, La Chine et sa nouvelle diaspora : la mobilité au service de la puissance
}

\section{Zhipeng Li}

\section{OpenEdition}

\section{Journals}

Édition électronique

URL : https://journals.openedition.org/e-migrinter/351

DOI : 10.4000/e-migrinter.351

ISSN : 1961-9685

Éditeur

UMR 7301 - Migrinter

Édition imprimée

Date de publication : 15 octobre 2014

Pagination : 103-107

ISSN : 1961-9685

Référence électronique

Zhipeng Li, "Carine Pina-Guerassimoff, La Chine et sa nouvelle diaspora : la mobilité au service de la puissance », e-Migrinter [En ligne], 12 | 2014, mis en ligne le, consulté le 20 mai 2021. URL : http:// journals.openedition.org/e-migrinter/351 ; DOI : https://doi.org/10.4000/e-migrinter.351 
Note DE LECTURE

\section{Pina-Guerassimoff, Carine (2012) La Chine et sa nouvelle diaspora : la mobilité au service de la puissance, Paris, Ellipses, 237 p.}

\section{Li Zhipeng*}

$A \begin{gathered}\text { vec le développement } \\ \text { économique mondial, les liens } \\ \text { entre la politique et l'économie }\end{gathered}$ internationale deviennent de plus en plus étroits. Par ailleurs, les recherches réalisées sur la diaspora chinoise ${ }^{1}$ sont nombreuses, et envisagent la Chine comme pays d'origine ou d'émigration. À ce titre, certains chercheurs (Ma Mung, 2000; Li, 2002) affirment qu'il faut envisager la diaspora chinoise comme une ressource, puisque ses membres ont toujours entretenu des liens affectifs, culturels ou encore économiques avec leur pays natal. La diaspora chinoise a en effet grandement contribué au développement économique de la Chine après le lancement des politiques d'ouverture et de réforme et actuellement, les thématiques de recherche portant sur la diaspora chinoise se concentrent principalement sur les réseaux économiques du monde chinois, le management et le développement des entreprises de ces migrants placés sous l'influence de la culture chinoise (Li, 2003). À l'inverse, d'autres

\footnotetext{
*L'auteur remercie Mélanie Pénicaud pour sa relecture attentive et ses précieux conseils.

${ }^{1} \mathrm{Il}$ existe un débat sur le bien-fondé d'utiliser la notion de diaspora à propos des Chinois d'outre-mer - voir notamment Ma Mung (2002, pp. 21-24) qui souligne qu'en chinois, la situation est différente : il n'y a pas, semble-t-il, d'équivalent du terme « diaspora ». Les différentes expressions qui désignent les populations d'origine chinoise établies hors de Chine ont fait et font encore l'objet de nombreuses discussions et surtout d'enjeux politiques. Ici, je renvoie, par l'expression « diaspora chinoise » à ce qui semble équivalent au sens général des «Chinois d'outre-mer » lorsque l'on parle des migrants chinois. Carine Pina-Guerassimoff ne souligne pas ces différentes expressions dans son ouvrage.
}

approches se situant du point de vue des pays d'accueil de la diaspora chinoise estiment que ces Chinois ne peuvent être perçus comme une expansion de la Chine (Li, 2003). Ainsi, toujours d'après ces mêmes chercheurs, c'est du point de vue de l'insertion à la société locale, à l'intégration et à l'assimilation qu'il convient d'étudier cette diaspora chinoise.

Ainsi, les recherches de Carine PinaGuerrassimoff, sinologue et politologue, portent spécialement sur les migrations internationales chinoises et proposent une nouvelle approche dans le champ des études sur cette diaspora. En effet, si la chercheuse a déjà publié de nombreux articles et ouvrages dans ce domaine (cf. notamment L'État chinois et les communautés chinoises d'outremer, 1997), elle vient néanmoins, avec cet ouvrage intitulé La Chine et sa nouvelle diaspora: la mobilité au service de la puissance, combler un vide important, en démontrant le lien économique qu'entretient depuis les années 1980, la Chine avec sa diaspora.

Cet ouvrage s'inscrit donc dans la lignée des recherches sur la nouvelle diaspora chinoise, en présentant et analysant la situation des "nouveaux migrants chinois $»^{2}$ depuis le lancement et la mise en place de la politique de réforme économique et d'ouverture dans les années 1980. L'auteure s'appuie sur une connaissance de la politique chinoise riche en informations chiffrées et données concrètes, surtout

\footnotetext{
${ }^{2}$ Xinyimin: "Les nouveaux migrants chinois». Ils regroupent l'ensemble des Chinois et de leurs enfants partis du pays depuis 1978 .
} 
puisées dans les sources chinoises (journaux officiels, articles scientifiques, lois, etc.) démontrant la manière dont le renouveau migratoire chinois participe de l'internationalisation du pays, non seulement sur le plan économique mais aussi social. Afin de souligner les éléments politiques favorables au développement économique et à la réunification nationale, l'auteure en vient à qualifier la politique d'affaires des Chinois de «politique diasporique». Elle montre ainsi en quoi cette mobilité de la diaspora chinoise sert la mondialisation économique de la Chine.

Afin de mieux traduire les relations entre la Chine et sa nouvelle diaspora, Carine Pina-Guerrassimoff divise son développement en trois grandes parties : en premier lieu, elle présente un changement politique à l'égard de l'émigration chinoise. Puis, elle met en lumière l'évolution de la "politique diasporique » chinoise à travers trois caractères: une administration spécifique, une promotion culturelle (l'association, les médias, la langue et la culture) et les intérêts de la diaspora chinoise. La troisième partie de cet ouvrage traduit l'idée que la migration est un atout du développement économique et de la réunification nationale. L'auteure présente en outre la situation du développement des migrants chinois en Afrique et en Amérique latine.

Dans la première partie de l'ouvrage, l'auteure montre que les conditions de départ des Chinois se sont améliorées durant ces deux dernières décennies, c'est-à-dire que les conditions de sortie du territoire sont devenues plus libres. On reconnait généralement trois périodes majeures du contrôle migratoire: de 1949 à 1977, les autorisations de sortie (et d'entrées) sont très limitées (p. 20), le franchissement des frontières est non plus seulement un acte politique, mais aussi un acte éminemment idéologique; de 1978-1986, les autorisations de sortie du territoire sont principalement réservées aux «affaire[s] publique[s]», et sont surtout délivrés des passeports diplomatiques (p. 21); enfin l'hypothèse d'une «affaire privée » se confirme par l'extension des possibilités de sortie du territoire à l'ensemble des citoyens entre 1987 et 2011 (p. 22). Cette dernière se traduit par le changement d'organisation de la mobilité du service public au marché privé, à savoir que « la consommation de la mobilité » (p. 25) a connu un développement avec l'amélioration de l'institutionnalisation des affaires de la diaspora chinoise. Par exemple, «la migration internationale chinoise est tout à fois facilitée et développée grâce à une offre croissante et diversifiée d'information, d'acteurs et de services liés à la mobilité. » (p. 25). En outre, l'auteure met en lumière une incitation officielle incarnée (p. 33) par le mythe de $H_{\text {Huaqiao }}{ }^{3}$ (p. 34) et l'engouement pour les Xinyimin (p. 35) dans la mobilité internationale chinoise: concrètement, si la mobilité permet d'accéder au capital économique, elle est aussi en Chine une garante de la reconnaissance sociale et politique. Ainsi les autorités n'encouragent pas ouvertement les départs, mais la singulière reconnaissance publique de ces migrants favorise l'engouement pour la migration internationale. Les Xinyimin sont décrits par les autorités comme éduqués, voire très qualifiés; doués et ambitieux. Ils sont par exemple, étudiants, entrepreneurs, artistes, ouvriers, salariés et cadres d'entreprises, professeurs, ont conservé leur nationalité chinoise, ou bien en ont changé récemment (p. 35).

La course à la mobilité des étudiants chinois et les circulations mercantiles favorisées (dans le domaine des affaires et $\mathrm{du}$ tourisme) se manifestent par un phénomène migratoire exemplaire. L'ouvrage s'arrête également sur les

\footnotetext{
${ }^{3}$ Huaqiao: les chinois résidants temporairement à l'étranger. En ce qui concerne le «mythe de Huaqiao", pour le dire simplement, les Huaqiao sont généralement très riches et incarnent la réussite parmi les non-migrants dans la société des années 1980, ce qui provoque un rêve d'émigration.
} 
mobilités chinoises illégales, une tendance décroissante et combattue, mais demeurant un phénomène international. Carine PinaGuerassimoff s'appuie ici sur les analyses de bases de données démontrant la croissance quantitative et la diversification des destinations.

Quant aux catégories de la migration chinoise, l'auteure montre que les migrants originaires des provinces littorales (Zhejiang, Guangdong et Fujian) sont liées principalement aux activités économiques, ce sont ceux qui «font des affaires». À l'inverse, les nouveaux migrants économiques appartiennent à la masse prolétaire et ont en commun leur absence, $a$ priori, de réseaux migratoires par rapport aux migrants de Zhejiang, de Guangdong et de Fujian, c'est-à-dire que les «nouveaux » migrants ne viennent pas de régions ayant une tradition migratoire, ne bénéficient pas des réseaux d'accueil ni de prise en charge familiale ou communautaire. L'auteur souligne, par ailleurs, qu'il existe un phénomène croissant de migration des élites, présentant une qualification supérieure, répondant à des offres d'emploi très sélectives, faisant leurs études à l'étranger, mais souvent boursiers ou autofinancés.

Carine Pina-Guerrassimoff dévoile également un aspect de la migration féminine à travers le cas de femmes partant seules, hors des cadres conventionnels (famille ou milieu étudiant), et poussées par la recomposition du marché international de la main d'œuvre de ces trois dernières décennies (p. 71). Elle analyse les raisons de départs de ces Chinoises et nous dévoile leurs motivations particulières, la raison de leur mobilité est une réponse à une demande internationale de main d'œuvre féminine émanant de secteurs économiques qui ne peuvent pas être délocalisés. Elles sont ainsi, domestiques pour les familles de Chinois entrepreneurs, serveuses pour les restaurants ethniques, femmes de chambre dans les hôtels, cumulent les petits boulots et certaines s'adonnent, de manière complémentaire ou principale, à des activités de prostitution. Par ailleurs, les Chinoises sont aussi bien employées dans les usines textiles d'Europe de l'Est et dans d'autres activités entrepreneuriales et commerciales. De plus, la migration pour mariage a toujours été une importante cause de la mobilité des femmes. Enfin, la réduction importante des perspectives professionnelles et économiques en Chine est une motivation majeure de leur projet migratoire, selon Carine Pina-Guerrassimoff.

La deuxième partie de l'ouvrage tente d'analyser la «politique diasporique» chinoise. Dans un premier temps, Carine Pina-Guerrassimoff s'attache à développer les pratiques administratives des institutions nationales et provinciales en charge de la diaspora chinoise depuis 1978. Elle présente ainsi les fonctions et les objectifs du Bureau des affaires des Chinois d'outre- $\mathrm{mer}^{4}$, de la Fédération nationale des Chinois d'outremer de retour ${ }^{5}$ ainsi que du parti politique du Zhi Gong Dang représentant à la fois la diaspora chinoise de retour et les familles d'émigrés. En outre, elle démontre que la réforme de ces trois principales institutions s'applique à celles chargées de la diaspora chinoise par l'amélioration de la formation des cadres et fonctionnaires, l'incitation du développement des moyens de communication de l'administration et l'image des pratiques développées au sein

\footnotetext{
${ }^{4}$ Bureau des affaires des Chinois d'outre-mer: organisation «verticale» en lien avec d'autres institutions mais qui n'a pas le rang ministériel. Il élabore et met en application les plans généraux de travail et les politiques concernant ces expatriés. Il traite de la protection des droits en Chine et des intérêts de la diaspora chinoise de retour ou des familles d'émigrés.

${ }^{5}$ Fédération nationale des Chinois d'outre-mer de retour : organisation « horizontale » dont les relais à l'étranger sont les associations des Chinois d'outremer. Son objectif est de créer des liens avec la diaspora chinoise et leurs organisations, développer l'amitié et promouvoir la coopération et les échanges. Elle opère sous la direction du Parti Communiste Chinoise (PCC). Des représentations locales et provinciales prolongent sa politique aux différents échelons administratifs.
} 
des autres administrations. Par exemple, les autorités cherchent à améliorer leurs connaissances des communautés expatriées et des Chinois de retour. Un groupe d'experts associant politiques et chercheurs a été créé au sein du Bureau des affaires d'outre-mer. Il en existe un semblable au sein de la Conférence Consultative Politique $\mathrm{du}$ Peuple Chinois (CCPPC) ${ }^{6}$. Des appels d'offres sont régulièrement lancés pour des études sur les communautés expatriées (p. 94).

Afin de fournir les informations et les contacts nécessaires aux entreprises qui souhaitent s'implanter à l'étranger, les services commerciaux et économiques jouent un rôle important, qui permet de comprendre le lien entre les administrations centrales, provinciales ou locales dans un système institutionnel transversal, spécifiquement chargé des communautés chinoises d'outre-mer. Ceci implique que les administrations nationales ou locales sont parties prenantes des questions relatives aux Chinois d'outre-mer. La chercheuse pose ici trois assertions: l'association comme intermédiaire jouant un rôle majeur dans le maintien des liens entre les émigrés et leurs régions d'origine; les médias comme support fondamental au sein des communautés émigrées chinoises pour servir aux Chinois d'outre-mer; et la langue et la culture comme enjeux sous-tendant l'existence des deux premiers, cette troisième affirmation étant au cœur du développement de l'éducation par les migrants dans leur société d'accueil et dans leur région d'origine. L'ensemble de ces points participe à protéger les intérêts des Chinois d'outremer à l'étranger. En effet, le droit à la nationalité est au fondement du lien avec le pays d'origine. Cette partie permet de connaitre le principal objectif des politiques diasporiques, qui se manifeste par le maintien du lien entre l'espace de départ et les migrants dans leurs États d'arrivée, ce qui revient à dire que «la politique chinoise du

${ }^{6}$ L'organe consultatif politique suprême de la Chine. lien est élaborée et mise en œuvre au cœur de l'État» (p. 91).

La dernière partie de l'ouvrage permet de connaitre le lien entre des communautés expatriées et la Chine à partir du point de vue de la "politique diasporique », à travers l'analyse des stratégies politiques et économiques. Concrètement, la migration est un atout du développement économique, auquel les remises familiales, les investissements entrepreneuriaux et les transferts de haute technologie participent. D'autre part, les migrants s'avèrent être un atout pour la réunification nationale; à ce propos, Carine Pina-Guerrassimoff discute principalement des problèmes politiques entre Pékin et Taïwan. L'auteure montre que les communautés chinoises anciennes et nouvelles jouent un rôle majeur dans les synergies qui façonnent les relations sinoaméricaines (par exemple, technologies, financements, et marchés sont introduits par les migrants de chaque côté du Pacifique. p. 203) et constate que c'est par «une présence quotidienne et massive de migrants chinois en terres africaines que s'illustre la nouvelle configuration des relations sinoafricaines» (p. 204). Cette partie, à propos des agents transnationaux de la puissance chinoise, démontre que l'émigration, ou encore aujourd'hui la mobilité, est mise au service des intérêts du pays, qu'ils soient nationaux ou internationaux.

Certes, la situation économique, sociale et politique de la Chine contribue à générer un accroissement des départs de Chinois à l'étranger, mais la politique d'immigration des pays d'accueil joue quand même un rôle inévitable pour inciter à la mobilité internationale. Selon toute logique, l'auteure n'a pas souligné cet aspect dans son livre, car c'est se placer du point de vue occidental, néanmoins, dans la conclusion de l'ouvrage, l'auteur ouvre à la réflexion un aspect crucial du complexe migratoire chinois à partir de l'approche occidentale : «En effet, les flux internationaux de population ont par le passé accompagné 
l'expansion d'États (européens) et fait l'objet de nombreuses théories. S'agit-il dans le cas chinois d'une redéfinition des colonies de peuplement, d'un nouveau modèle de colonie de comptoir ou bien d'un avatar de la conception impériale chinoise ? » (p. 237).

L'ouvrage de Carine PinaGuerrassimoff permet de nous poser les questions relatives à un schéma des relations entre des phénomènes migratoires transnationaux et la puissance du pays d'origine. La connaissance approfondie de la dynamique migratoire de la «nouvelle diaspora » chinoise que nous livre ce riche ouvrage fournit au lecteur de nouvelles clés de compréhension de la Chine à travers sa diaspora.
李明欢 LI Minghuan (2002) 年8月；08， 2002，欧洲华侨华人史 $A$ bistory of Chinese Immigrants in Europe, 北京Beijing, 中国华侨出版社 Maison d'édition de Huaqiao de Chine, 860 p.

李安山 LI Anshan

\section{中国华侨华人研究的历史与现状概述}

Aperçu de l'bistoire et de la situation actuelle des recherches sur les Chinois d'outre-mer, 中国日报China Daily，2003年8月8日， 08/08/2003.
Li Zhipeng

Doctorant en géographie

MIGRINTER-UMR7301

CNRS/Université de Poitiers zhipeng.li@univ-poitiers.fr

\section{Bibliographie}

Ma Mung, Emmanuel (2000) La diaspora chinoise: géographie d'une migration, Paris, Ophrys, 175 p. (GéOphrys).

Ma Mung, Emmanuel (2002) La désignation des diasporas sur Internet, Hommes et Migrations, $\mathrm{n}^{\circ} 1240$, pp. 19-28. 